Pacific Journal of Mathematic 


\title{
ON STRICTLY SEMI-SIMPLE BANACH ALGEBRAS
}

\author{
EDITH H. LUCHINS
}

I. Introduction. Define the strict radical of an algebra to be the intersection of just those of its two-sided ideals which are regular maximal right ideals. Call the algebra strictly semi-simple (sss) if its strict radical is the zero ideal. This note proves that the strict radical of a real Banach algebra $B$ contains the set of topologically nilpotent elements of $B$. Also, it gives a condition which is both necessary and sufficient for $B$ to be sss.

II. Preliminaries. For any ring or algebra $A$ let $T(A)$ denote the set of all those two-sided ideals in $A$ which are regular maximal right ideals. The intersection of the elements of $T(A)$ is the strict radical of $A . A$ is strictly semi-simple (sss) if its strict radical is the zero ideal.

Lemma 1. Let $I$ be a two-sided ideal in the algebra (ring) $A$. Then the following are equivalent:

(a) $I \in T(A)$, that is, $I$ is a regular maximal right ideal.

(b) I is a regular maximal left ideal.

(c) $A / I$ is a division algebra (division ring).

Proof. Use is made of the theorem [4, Theorem 24.6.1] that a division algebra has no proper right or left ideals and that an algebra with no proper right ideals either is trivial or is a division algebra.

If (a) holds, then $A / I$ has no proper right ideals. Now $A / I$ is not trivial since if $j$ is a left unit element of $A$ modulo $I, j^{\prime} \cdot j^{\prime}=j^{\prime} \neq 0$ (where $x^{\prime}$ denotes the image of $x \in A$ under the canonical homomorphism of $A$ onto $A / I$ ). The cited theorem shows $A / I$ is a division algebra. Thus (a) implies (c) and, similarly, (b) implies (c). Moreover, if (a) holds, then $j^{\prime}$ is a left identity for $A / I$ and hence an identity for it, so that $I$ is regular with $j$ as its associated unit element. If $I \subset L, L$ a left ideal in $A$, then $L / I$ is a left ideal in $A / I$, and an improper ideal by the cited theorem, so that $L=I$ or $A$ and $I$ is a regular maximal left ideal. Thus (a) implies (b).

Suppose (c) holds and $e^{\prime}$ is a unit of $A / I$. Then $I$ is regular with

Received August 5, 1958, in revised form December 1, 1958. This report was prepared while the author held the New York State Fellowship, 1957-8, of the American Association of University Women. It is based in part on the author's doctoral dissertation written at the University of Oregon under the sponsorship of Professor Bertram Yood. Thanks are extended to the referee for helpful suggestions. 
$e$ as its associated unit element. If $I \subset J, J$ a right ideal in $A, J / I$ is a right improper ideal in $A / I$ so that $J=I$ or $A$ and $I$ is a regular maximal right ideal. Thus (c) implies (a).

Theorem 1 relates the strict radical of $A$ to the Jacobson radical [5] and to the Segal [9] or Brown-McCoy radical [2], which is the intersection of the regular maximal two-sided ideals in $A . A$ is called strongly semi-simple (semi-simple) if the Segal (Jacobson) radical is the zero ideal.

A satisfies Property $M$ if each of its regular maximal right ideals is a two-sided ideal.

THEOREM 1. The strict radical contains the Segal and Jacobson radicals so that if $A$ is sss then it is necessarily strongly semi-simple and semi-simple. These radicals coincide if Property $M$ is satisfied.

Proof. Let $W$ be the set of all regular maximal two-sided ideals and $W_{r}$ the set of all regular maximal right ideals in $A$. If $I \in T(A)$ then $A / I$ is a division algebra by Lemma 1 so that $I \in W$. Therefore the strict radical contains the Segal radical which contains the Jacobson radical. Now, if Property $M$ holds, $I \in W_{r}$ implies $I \in T(A)$ which shows the Jacobson radical contains the strict radical and hence all these radicals coincide.

EXAMPLES. 1. An example of an algebra which is semi-simple and strongly semi-simple but not sss is furnished by the algebra of all 2 by 2 matrices, which is radical in the sense of the strict radical.

2. Arens' $B Q^{*}$-algebra [1] are examples of Banach algebras which are sss and satisfy Property $M$. Indeed, Arens establishes that such algebras are semi-simple and have the property that every closed idealand, a fortiori, every regular maximal right ideal-is two-sided.

3. Let $C(X, D)$ be the ring of all continuous functions on $X$ with values in $D$, where $X$ is a compact $T_{0}$-space and $D$ a division ring that admits a continuous function $f(x)$ such that $x f(x)+y f(y)=0$ implies that $x=y=0$. Kaplansky [6, p. 179] notes that such a function $f(x)$ cannot exist in a ring of characteristic 2 (take $x=y$ ) but exists in every ring of characteristic different from 2 that he has examined. The maximal right (or left) ideals in $C(X, D)$ are two-sided [6, p. 180], so that $C(X, D)$ satisfies Property $M$ and, since it is semi-simple, it is necessarily sss.

4. If a ring $A$ is strongly regular (that is, if for every $a \in A$ there exists $x \in A$ such that $a^{2} x=a$ ) then $A$ is semi-simple and every ideal in it is two-sided [2, pp. 462-4]. Hence a strongly regular ring satisfies Property $M$ and is sss. 
III. Necessary and sufficient condition for a Banach algebra to be sss. Henceforth the algebras considered are over the real field and the homomorphisms considered are algebraic (real-linear). Let $Q$ denote the quaternions, $H(A, Q)$ the set of nonzero homomorphisms of the algebra $A$ into $Q,|q|$ the absolute value of the quaternion $q$, and $C(X, Q)$ the algebra of quaternion-valued functions, continuous on and vanishing at the infinite point of a locally compact Hausdorff space $X$.

Lemma 2. An algebra $A$ is mapped onto the reals, onto a field isomorphic to the complexes, or onto the quaternions by any $h \in H(A, Q)$ and the kernel of $h$ belongs to $T(A)$. If $A$ is a Banach algebra each member of $T(A)$ is the kernel of some member of $H(A, Q)$.

Proof. Let $h(A)$ denote the image of $A$ under $h$. For any $u, v \in h(A)$

$$
|u \cdot v|=|u| \cdot|v| \text {. }
$$

Under the norm $|u|, h(A)$ is a normed algebra. A normed algebra in which the norm satisfies property (1) is isomorphic to either the reals, complexes, or quaternions [7, Theorem II]. Hence $A / h^{-1}(0)$ is a division algebra and $h^{-1}(0) \in T(A)$ by Lemma 1 .

Let $A$ be a Banach algebra and $I \in T(A)$. Then $A / I$ is a division algebra by Lemma 1 and a Banach algebra since $I$ is closed. A normed division algebra is isomorphic to the reals, complexes, or quaternions [7, Theorem I]. Hence $I$ is the kernel of some $h \in H(A, Q)$.

Theorem 2. Any subalgebra $A$ of $C(X, Q)$ is sss.

Proof. Let $f \in A, f \neq 0$. Then there is an $x \in X$ such that $f(x) \neq 0$. Let $I=\{g \in A: g(x)=0\}$. Then $A / I$ is naturally isomorphic to a subalgebra of $Q$. Hence $I \in T(A)$ by Lemma 2. But $f \notin I$. Therefore $A$ is sss.

Theorem 3. If a Banach algebra $B$ is sss, then $B$ is isomorphic with a subalgebra of some $C(X, Q)$.

Proof. Let $X=H(B, Q)$. There is a natural homomorphism of $B$ into $C(X, Q): f \rightarrow \varphi$ where $\varphi(x)=x(f)$. It remains only to show that the homomorphism is $1-1$. Let $f \in B, f \neq 0$. Since $B$ is sss there is an $I \in T(B)$ such that $f \notin I$. By Lemma 2, $I=x^{-1}(0)$ for some $x \in X$. Hence $\varphi(x) \neq 0$.

CoRollary 1. An algebra isomorphic to a subalgebra of a sss Banach algebra is itself sss. Hence any subalgebra, whether closed or not, of a sss Banach algebra is itself sss. 
IV. The strict radical of a Banach algebra contains the set of topologically nilpotent elements. An element $x$ of a normed algebra is called topologically nilpotent if $r(x)=0$ where $r(x)=\lim _{n \rightarrow \infty}\left\|x^{n}\right\|^{1 / n}=\sup |\beta|$ : $\beta \in$ spectrum of $x$ [8, pp. 617-618].

THeOREM 4. Let $N$ be the set of topologically nilpotent elements of a Banach algebra $B$ and $S$ the strict radical of $B$. Let $J^{\prime}$ be the Jacobson radical of any subalgebra of $B$. Then $J^{\prime} \subset N \subset S$.

Proof. That $J^{\prime} \subset N$ is known [8, Lemma 1.2]. If it is shown that every $h \in H(B, Q)$ maps $x \in N$ into the zero element, then it follows from Lemma 2 that $x$ belongs to every member of $T(B)$ and therefore to $S$. The spectrum of $h(x)$ contains the spectrum of $x$; hence $r[h(x)]=0$ since $r(x)=0$. Since a topologically nilpotent element is singular [4, p. 121], $h(x)=0$. Hence $N \subset S$.

COROLlary 2. If a Banach algebra is sss then zero is its only topologically nilpotent element.

Corollary 3. Let $N$ and $S$ be defined as in Theorem 4 and let $J$ be the Jacobson radical of $B$. Then $J=S$ if and only if $N=S$. If $B$ satisfies Property $M$, then $J=N=S$.

Proof. Theorem 4 yields Corollary 2 as an immediate consequence and also shows that if $J=S$, then $J=N=S$. If $N=S, N$ is an ideal composed of topologically nilpotent elements and therefore $N \subset J$ since $J$ is the union of such ideals [8, p. 617]; hence $J=N$. If Property $M$ is satisfied then $J=S$ by Theorem 1 so that $J=N=S$.

\section{REFERENCES}

1. R. Arens, Approximation in, and representation of, certain Banach algebras, Amer. J. Math. 71 (1949), 763-790.

2. __ and I. Kaplansky, Topological representation of algebras, Trans. Amer. Math. Soc. 63 (1948), 457-481.

3. B. Brown and N. H. McCoy, Radicals and subdirect sums, Amer. J. Math. 69 (1947), 46-58.

4. E. Hille and R. S. Phillips, Functional analysis and semi-groups, Amer. Math. Soc. Colloquium Publication vol. 31, Revised Edition, New York, 1957.

5. N. Jacobson, The radical and semi-simplicity for arbitrary rings, Amer. J. Math. 67 (1945), 300-320.

6. I. Kaplansky, Topological rings, Amer. J. Math. 69 (1947), 153-183.

7. S. Mazur, Sur les anneaux linéaires, C. R. Acad. Sci., Paris 207 (1938), 1025-1027.

8. C. E. Rickart, The uniqueness of norm problem in Banach algebras, Ann. of Math. 51 (1950), 615-628.

9. I. E. Segal, The group algebra of a locally compact group, Trans. Amer. Math. Soc. 61 (1947), 69-105. 


\section{PACIFIC JOURNAL OF MATHEMATICS}

\section{EDITORS}

\section{David Gilbarg}

Stanford University

Stanford, California

\section{R. A. Beaumont}

University of Washington

Seattle 5 , Washington

\author{
A. L. Whiteman
}

University of Southern California Los Angeles 7, California

L. J. Paige

University of California

Los Angeles 24, California

\author{
E. F. BECKENBACH \\ C. E. BURGESS \\ E. HEWITT \\ A. HORN
}

\author{
V. GANAPATHY IYER \\ R. D. JAMES \\ M. S. KNEBELMAN \\ L. NACHBIN
}

ASSOCIATE EDITORS
I. NIVEN

T. G. OSTROM

H. L. ROYDEN

M. M. SCHIFFER
E. G. STRAUS

G. SZEKERES

F. WOLF

K. YOSIDA

\section{SUPPORTING INSTITUTIONS}

\author{
UNIVERSITY OF BRITISH COLUMBIA \\ CALIFORNIA INSTITUTE OF TECHNOLOGY \\ UNIVERSITY OF CALIFORNIA \\ MONTANA STATE UNIVERSITY \\ UNIVERSITY OF NEVADA \\ OREGON STATE COLLEGE \\ UNIVERSITY OF OREGON \\ OSAKA UNIVERSITY \\ UNIVERSITY OF SOUTHERN CALIFORNIA
}

\author{
STANFORD UNIVERSITY \\ UNIVERSITY OF TOKYO \\ UNIVERSITY OF UTAH \\ WASHINGTON STATE COLLEGE \\ UNIVERSITY OF WASHINGTON \\ * * * \\ AMERICAN MATHEMATICAL SOCIETY \\ CALIFORNIA RESEARCH CORPORATION \\ HUGHES AIRCRAFT COMPANY \\ SPACE TECHNOLOGY LABORATORIES
}

Mathematical papers intended for publication in the Pacific Journal of Mathematics should be typewritten (double spaced), and the author should keep a complete copy. Manuscripts may be sent to any one of the four editors. All other communications to the editors should be addressed to the managing editor, L. J. Paige at the University of California, Los Angeles 24, California.

50 reprints per author of each article are furnished free of charge; additional copies may be obtained at cost in multiples of 50 .

The Pacific Journal of Mathematics is published quarterly, in March, June, September, and December. The price per volume (4 numbers) is $\$ 12.00$; single issues, $\$ 3.50$. Back numbers are available. Special price to individual faculty members of supporting institutions and to individual members of the American Mathematical Society: $\$ 4.00$ per volume; single issues, $\$ 1.25$.

Subscriptions, orders for back numbers, and changes of address should be sent to Pacific Journal of Mathematics, 2120 Oxford Street, Berkeley 4, California.

Printed at Kokusai Bunken Insatsusha (International Academic Printing Co., Ltd.), No. 6, 2-chome, Fujimi-cho, Chiyoda-ku, Tokyo, Japan.

PUBLISHED BY PACIFIC JOURNAL OF MATHEMATICS, A NON-PROFIT CORPORATION

The Supporting Institutions listed above contribute to the cost of publication of this Journal, but they are not owners or publishers and have no responsibility for its content or policies. 


\section{Pacific Journal of Mathematics}

\section{Vol. 9, No. $2 \quad$ June, 1959}

Lee William Anderson, On the breadth and co-dimension of a topological lattice

Frank W. Anderson and Robert L. Blair, Characterizations of certain lattices

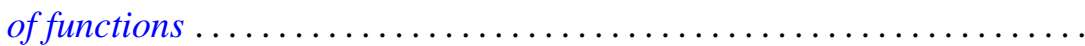

Donald Charles Benson, Extensions of a theorem of Loewner on integral

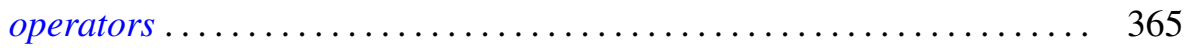

Errett Albert Bishop, A duality theorem for an arbitrary operator ........ 379

Robert McCallum Blumenthal and Ronald Kay Getoor, The asymptotic distribution of the eigenvalues for a class of Markov operators ........

Delmar L. Boyer and Elbert A. Walker, Almost locally pure Abelian

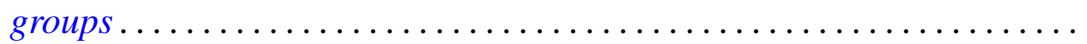

Paul Civin and Bertram Yood, Involutions on Banach algebras ........... Lincoln Kearney Durst, Exceptional real Lehmer sequences .... 415

Eldon Dyer and Allen Lowell Shields, Connectivity of topological

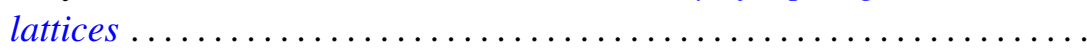

Ronald Kay Getoor, Markov operators and their associated

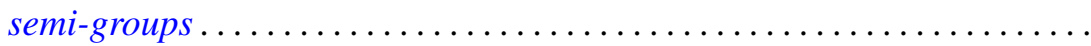

Bernard Greenspan, A bound for the orders of the components of a system of algebraic difference equations

Branko Grünbaum, On some covering and intersection properties in

Minkowski spaces ............................

Bruno Harris, Derivations of Jordan algebras ..............

Henry Berge Helson, Conjugate series in several variables.

Isidore Isaac Hirschman, Jr., A maximal problem in harmonic analysis.

II .

Alfred Horn and Robert Steinberg, Eigenvalues of the unitary part of a matrix

Edith Hirsch Luchins, On strictly semi-simple Banach algebras ...

William D. Munro, Some iterative methods for determining zeros of

functions of a complex variable...

John Rainwater, Spaces whose finest uniformity is metric .

William T. Reid, Variational aspects of generalized convex functions ....

A. Sade, Isomorphisme d'hypergroupoï des isotopes ...... . .

Isadore Manual Singer, The geometric interpretation of a special

connection . . .

Charles Andrew Swanson, Asymptotic perturbation series for characteristic

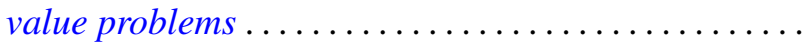

